Aim Haemophagocytic lymphohistiocytosis (HLH) is a rare, lifethreatening disorder. It can present to many specialities and individual Paediatricians are unlikely to develop significant experience of it. Early recognition and treatment improves the clinical outcome. The genetic basis of HLH is evolving and identification of viral triggers influencing management.

We aimed to study cases of HLH presenting to our tertiary Paediatric Hospital to identify the proportion of de novo cases and evaluate the role of Ebstein Barr virus (EBV) and genetic defects in the pathogenesis of HLH.

Methods Immunology, Rheumatology and Haematology Consultants identified children diagnosed with HLH over a 10 year period from 2003-2013. ICD10 codes were cross referenced. Case notes and electronic data were interrogated to extract relevant data.

Results 20 children were diagnosed with HLH over the 10 year period. Seven patients were de novo diagnoses and 13 had known haematological malignancy or rheumatological conditions.

At presentation, 15\% met the HLH 2004 diagnostic criteria, rising to $45 \%$ at time of diagnosis (Figure 1).

Viral triggers were investigated by EBV PCR and/or throat swab in 50\% of patients and all de novo HLH patients were EBV positive. Of those found to be EBV positive, Rituximab was given to 4 patients. Genetic mutations were looked for in $40 \%$ of all patients (Figure 2).

Nine patients recovered with standard management, 6 died and 5 proceeded to bone marrow transplant.

Conclusions HLH can present to a variety of paediatric specialities and a high index of suspicion is needed to make a timely diagnosis, as not all patients have 'classical' features at presentation. Identification of EBV gives the opportunity to use Rituximab effectively. In children with unusual responses to ubiquitous viruses, such as EBV, the competency of the host should be questioned. Some genetic defects predisposing to HLH in whom long term management with stem cell transplant can be curative are already known, and further defects may be discovered in the future. Accurate genetic diagnosis not only influences management, but is important for the families affected.

\section{G462(P) CAPTURING T-CELL RECEPTORS. A POTENTIAL NEW MODALITY FOR TARGETING HEPATIC TUMOURS AND POST-TRANSPLANTATION LYMPHOPROLIFERATIVE DISEASE (PTLD)}

${ }^{1,2} \mathrm{ND}$ Ruth, 'S Penny, 'L Steadman, 'D Millar, ${ }^{3} \mathrm{D}$ Hunt, ${ }^{3} \mathrm{P}$ Trantham, ${ }^{1,2} \mathrm{D}$ Kelly, ${ }^{1} \mathrm{M}$ Cobbold. 'School of Infection and Immunity, University of Birmingham Medical School, Birmingham, UK; ${ }^{2}$ Hepatology, Birmingham Children's Hospital NHS Foundation Trust, Birmingham, UK; ${ }^{3}$ Department of Chemistry, University of Virginia, USA

\subsection{6/archdischild-2015-308599.416}

Background The immune system plays an important part in recognising infection and diseases including cancer. Malignant cells express specific proteins on their cell surface. It is widely believed that it is these proteins that the immune system uses to recognise tumours and eventually eradicate them. When this process goes wrong, a tumour forms. The immune response to cancers in general is widely accepted to play an important role

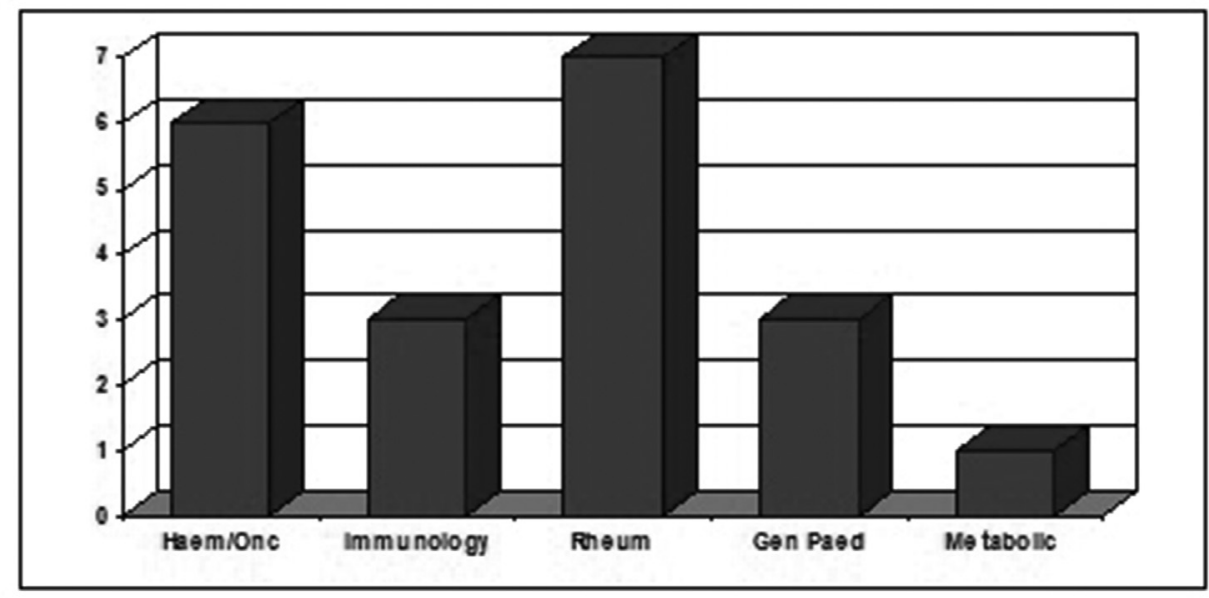

Abstract G461(P) Figure 1 Presenting specialities of children diagnosed with HLH

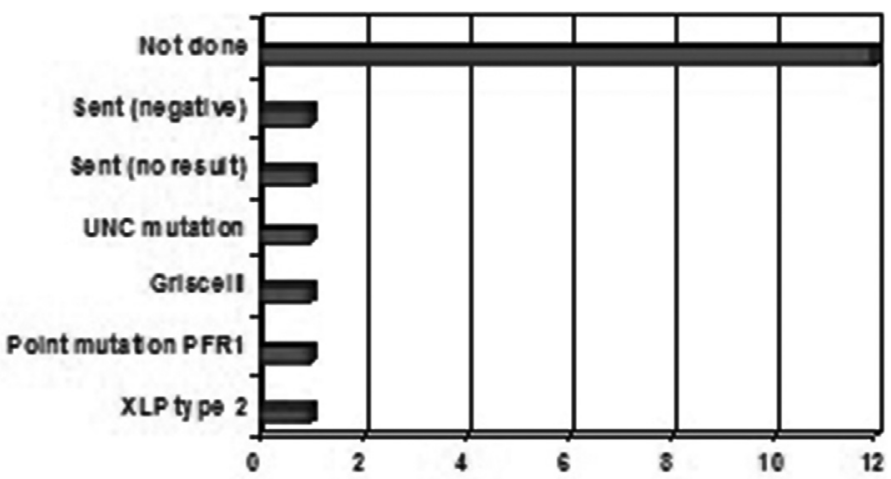

Abstract G461(P) Figure 2 Genetic studies in children with HLH 
in control of tumorigenic processes, by using tumour infiltrating lymphocytes. How this process works is still being established, however there remains a potential target for cancer specific immunomodulatory treatment regimens.

Aim (1) To identify tumour specific MHC class I phosphopeptide antigens on lymphoblastoid cell lines LCL's (an in vitro model for PTLD) as well as hepatic tumour tissues. (2) T-cells are immune cells which are notoriously difficult to maintain in long-term culture and as a result it is difficult to establish an 'off the shelf' T-cell product, however the aim of this project was to explore potential modalities for capturing the T-cell receptor (TCR), important in recognising tumour specific antigens and the resultant product could be used to establish a non patientspecific, but tumour specific product.

Patients and methods Paediatric and adult patients were identified with hepatic malignancy and consented as per current policy. Cells were isolated and tumour specific phosphopeptide antigens were identified. These provide the targets for T-cells, and more specifically TCR's. Having identified these antigens, modalities have been explored for expanding these cells. Hybridoma technology is long established in immortalising B-cells, and this study aims to explore its potential with immortalisation of T-cells.

Results A number of novel phosphopeptide antigens have been identified both in vitro as well as on patient tissues. This information has been used to identify potential T-cell targets and by formation of hybridomas we have established a method for expanding specific T-cell's in vitro. Although these hybridomas are currently unstable due to their tetraploid status, we aim to modify this protocol further to allow for stable expansion of hybridoma cells which possess the relevant TCR motif.

Conclusions Identifying a modality for expanding cells with a specific TCR repertoire clearly allows us to target tumour specific phosphopeptide antigens and has the potential to be developed as an immunomodulatory therapy in patients with hepatic tumours or PTLD.

\section{G463(P) DETERMINING THE HEALTH NEEDS OF CHILDREN WITH SPECIAL EDUCATIONAL NEEDS}

${ }^{1} \mathrm{H}$ Miller, ${ }^{1} \mathrm{~T}$ Juniper, ${ }^{1} \mathrm{P}$ Reid, ${ }^{1} \mathrm{C}$ Lloyd, ${ }^{2} \mathrm{~A}$ Goodson, ${ }^{2} \mathrm{M}$ Rose. ${ }^{1}$ Medical School, Oxford University, Oxford, UK; ${ }^{2}$ Community Paediatrics, Oxford University Hospitals Trust, Oxford, UK

\subsection{6/archdischild-2015-308599.417}

Introduction The goal of the SEND reforms is to improve the outcomes of children with special educational needs (SEN) and disabilities. One key strategy is to improve the coordination and integration of education, health and care services through joint commissioning. Commissioning can only be effective when services are designed around the needs of their services users. Little is known about the health needs of disabled children for whom the lack of data for planning purposes is the single biggest challenge to better outcomes.

\section{Aim}

1. To describe the health problems and services use of children with SEN.

2. To pilot an approach to data collection for services planning.

A third objective, to assess the quality of medical reports, is reported separately.
Method We reviewed all the medical reports submitted between January and June 2014 for a Statement of Special Educational Needs (SSEN). Additional hospital statistics were collected.

Results Data were analysed on 125 children, 78\% boys and $22 \%$ girls, median age 6.9 years (1.9-14.8). 65\% of children were known to community paediatrics and $25 \%$ to CAMH services. $6 \%$ of children were LAC, $5 \%$ on CP plan, and $15 \%$ excluded from school.

$39 \%$ children had autism, 7\% ADHD, 26\% SEMH, 9\% physical needs, 8\% sensory impairment (all types), 26\% SLCN, 46\% learning difficulties (all types), 8\% SpLDs. 55\% of the children had multiple needs, $6 \%$ were complex special needs.

In the previous year $62 \%$ children attended hospital outpatients (1-16 visits), $10 \%$ of children were admitted to hospital (1-4 admissions) and $23 \%$ of children had been seen in A\&E (1-7 visits). 50\% of children were known to hospital specialities, $50 \%$ to S\&L, 16\% physiotherapy, 30\% OT services.

Many children had associated health problems (19\% medical, 18\% nutrition, 9\% continence).

Conclusions Children with SEN have extensive health needs and are high users of health services. Our data is likely to be an underestimate as we only analysed hospital statistics and medical reports whose quality varied.

SSEN reports provide important health data. Data collection and analysis from a broader range of services continues. The new Education, Health and Care process provides an opportunity for prospective data collection for services planning.

\section{G464(P) TIME TO DISCUSS PREVENTION STRATEGIES FOR GROUP B STREPTOCOCCUS DISEASE}

S Salehian, A Rastogi, A Fraisse, 0 Ghez, M Burmester. Paediatric Intensive Care Unit, The Royal Brompton Hospital, London, UK

\subsection{6/archdischild-2015-308599.418}

Aims Group B Streptococcus (GBS) can cause neonatal septicaemia, meningitis and rarely, infective endocarditis. Through this case study, we aim to raise awareness of the devastating sequelae of GBS disease and the importance of early recognition of complications.

Case discussion: A term infant born in good condition was being managed by the community midwifery team for feeding difficulties, failing to thrive and presented to A\&E twice with lethargy and maternal concern. At two weeks of age he represented having additionally developed fever and was admitted and treated for GBS septicaemia and meningitis. He was discharged after 5 days to ambulatory care for continuation of antibiotics. 10 days into treatment his mother became worried and he presented to A\&E in cardiogenic shock with a heart murmur, enlarged liver and rising lactate.

Once transferred to the Paediatric Intensive Care Unit, he required ventilation and inotropic support for poor cardiac output, and conservative medical treatment for necrotising enterocolitis, presumed secondary to embolic pathology. An echocardiogram demonstrated two large, mobile masses attached to the mitral valve leaflets with severe mitral regurgitation and right ventricular dysfunction, consistent with infective endocarditis in an otherwise structurally normal heart (Figure 1). Surgical repair of his valve was not possible and so a $17 \mathrm{~mm}$ prosthetic St Jude's Valve was placed in the mitral position. Post-operatively he developed a low cardiac output state, seizures, and 